Verfügung stehen, welche die Glaubwürdigkeit forensisch prüfbar machen. Schließlich stehen aber auch noch die klassischen forensischen Glaubwürdigkeitskriterien, wie sie von Steller, Köhnken und Volbert dargestellt wurden, zur Verfügung. Hier werden Darstellungsverhalten, inhaltlicher Kontext und Einbindung des Inhalts in den zeitlich-örtlichen Rahmen auf ihre Stimmigkeit und Glaubwürdigkeit untersucht. Dieses Instrument stellt im Rahmen der Begutachtung in Asylverfahren eine wertvolle Matrix dar. Jedoch sind auch die Rahmenbedingungen für die Exploration von Asylbewerbern von wesentlicher Bedeutung. Daher sollen sie im Folgenden kurz zusammengefasst werden:

\section{Unerlässliche Voraussetzungen für die Begutachtung trau- matisierter Asylwerber}

- Vertrauensbildung durch Herstellung eines respekt- und verständnisvollen Umgangs.

- Möglichst: Gleichgeschlechtlichkeit des Gutachters bzw. der Gutachterin.

- Absolut: Gleichgeschlechtlichkeit des Dolmetschers bzw. der Dolmetscherin.

- Ausreichend Zeit für die Exploration.

- Beachtung und Berücksichtigung des sozialen Kontexts zur Zeit der Befragung.
Zusammenfassend muss davon ausgegangen werden, dass eine Vielzahl von Faktoren unumgänglich beachtet werden muss, wenn traumatisierte Asylbewerber einer Begutachtung unterzogen werden. Voraussetzung ist, dass Gutachter und Behörden über ausreichendes Wissen über den soziokulturellen, ethnischen und religiösen Hintergrund und die psychische Situation von Flüchtlingen mitbringen und über eine ausreichende humanitäre Einstellung verfügen, die sie befähigt, sich auf die oft stark belastenden Inhalte ohne die Entwicklung einer Abwehr als Selbstschutz einzulassen.

Wie bei vielen wichtigen Dingen im Leben gilt auch hier das Prinzip, dass nur wer sich vorurteilslos und respektvoll auf die Wahrheit einlässt, mit ihr belohnt wird.

\section{Literatur}

Berger: Psychiatrie und Psychotherapie, München-Wien-Baltimore, 1999 ICD 10, Kapitel V (F), klinisch-diagnostische Leitlinien, WHO. Hrsg. von H. Dilling, W. Mombour, M.H. Schmidt, E. Schulte-Markwort, 2. Aufl. H. Huber Bern, Göttingen; Toronto: 2000

Steller, M. Volbert R. (1999). Forensisch-aussagepsychologische Begutachtung (Glaubwürdigkeitsbegutachtung). In: Praxis der Rechtspsychologie, 9 (2), 46-106.

〈http://www.unhcr.de/laenderinformationen.html> (Zugriff: 28. 10. 2010) <http://www.bbc.co.uk/archive/collections.shtml〉 (Zugriff: 28. 10. 2010)

\title{
Praxis des interkulturellen Trainings in der Justiz
}

\section{Prof. Dr. Christiane J. Driesen}

Fachbereich Kommunikation und Medien, Hochschule Magdeburg-Stendal (FH)

\section{Eine Herausforderung}

Das zu behandelnde Thema empfand ich zuerst als eine Herausforderung: Als Dolmetscherin und auch Ausbilderin von juristischen Dolmetscher(inne)n und Übersetzer(inne)n bin ich zwar für die Justiz tätig, es ist jedoch als Nicht-Mitglied des Justizwesens unmöglich, über eine erschöpfende Kenntnis aller zur Zeit eventuell laufenden Projekte zu verfügen. Im Rahmen meiner Beteiligung an einigen Initiativen der EU-Direktion für Mehrsprachigkeit und Dolmetschen (Reflection Forum on Multilingualism and Interpreter Training $)^{1}$ und Projekten der EU-Direktion für Justiz insbesondere des Forums „Criminal Justice“ und der Ausschreibungen² dieser Generaldirektion im Rahmen des Justizprogramms ist mir jedoch deutlich geworden, dass das Bewusstsein für eine notwendige Öffnung auf die zumindest in den anderen EU-Staaten herrschenden unterschiedlichen gerichtlichen Usancen stärker wird. Einige Programme befassen sich mit der Arbeit mit Dolmetscher(inne)n. Die am 16. Juni 2010 verabschiedete EURichtlinie zum Recht auf Dolmetschleistungen und Übersetzungen im Strafverfahren wird sicherlich entscheidende Änderungen mit sich bringen.

\section{Inwieweit sollte die Justiz sich einem interkulturellen Trai- ning unterziehen?}

Die aufgestellte Frage kann provokativ klingen: Entscheidungsträger der Justiz sind mit immer komplexer werdenden Aufgaben betraut. Da sie meist in einem bestimmten Land tätig sind und einem bestimmten Rechtssystem dienen, kann von ihnen wohl nicht verlangt werden, dass sie sich in die Rolle eines Kultursachverständigen begeben. Sollte von ihnen die Offenheit für fremde Kulturen qua Beruf verlangt werden? Es ist noch nicht der Fall, jedoch könnte eine solche Offenheit ihre Aufgaben bedeutend erleichtern. Indem sie zu einer erfolgreichen Kommunikation beiträgt, ist die Offenheit für fremde Kulturen ein Garant der Gleichheit aller Menschen (Verdächtige, Angeklagte, Zeug(inn)en, Opfer) vor dem Gesetz. ${ }^{3}$ Dadurch kann rechtliches Gehör tatsächlich gewährleistet und zur Wahrheitsfindung effizienter beigetragen werden.

Darüber hinaus wird die Offenheit für fremde Kulturen notwendiger in einer Zeit, in der die nationalen Gerichte auch EU-Recht anwenden müssen und immer häufiger in Kontakt

\footnotetext{
1 <http://www.europarl.europa.eu/sides/getDoc. do?type $=$ TA\& reference $=P 7-T A-2010-0220$ \&language $=D E \&$ ring $=A 7-2010-0198>$ (Zugriff 14.10.2010).

2 <http://ec.europa.eu/justice/funding/jpen/call_20100611/ag call 2010 en.pdf> (Zugriff 14.10.2010).

3 EMRK Art. 6, GG Art. 3.1, 3.3.
} 
mit ihren Kolleg(inn)en aus anderen Ländern kommen. Eine interkulturelle Sensibilisierung wäre daher durchaus sinnvoll.

\section{Warum „lediglich“ eine interkulturelle Sensibilisierung?}

Heutzutage wird die Justiz europaweit mit vielen unterschiedlichen Kulturen konfrontiert. Die in Europa vorkommenden Kulturen variieren entsprechend der einzelnen EU-Mitgliedstaaten und der oft wechselnden Migrant(inn)enwellen, die alte und neue Krisenherde verlassen.

Da Kultur und Sprachen offensichtlich die beiden Seiten derselben Medaille bilden, können sich die juristischen Berufe kaum mit jeder neuen, plötzlich in den Vordergrund tretenden Kultur gründlich auseinandersetzen, und aus diesem Grunde kann „lediglich“ eine Sensibilisierung angestrebt werden. Im Folgenden wird gezeigt, dass dieses Bestreben für die gerichtlichen Entscheidungsträger(innen) durchaus effizient sein kann und zum gezielten Einsatz vertrauenswürdiger Kultursachverständiger bzw. Dolmetscher(innen) führen sollte.

\section{Auf welche interkulturellen Kernunterschiede 4 sollte die Sensibilisierung besonders abzielen?}

Insbesondere bei bizarr anmutenden oder gar schockierenden Ausführungen sollten die aufgeführten Kernunterschiede in der interkulturellen Kommunikation systematisch beachtet werden. Diese sollen mit kurzen Beispielen im Folgenden illustriert werden.

\section{Zeitgefühl}

Je nach Kultur ist das Wahrnehmen von Zeitspannen unterschiedlich. Ein Augenblick im Deutschen entspricht zum Beispiel kaum dem „un momentito“ im mexikanischen Spanisch. In Deutschland ist es üblich, um 20 Uhr zu erscheinen, wenn die Einladung zum Abendessen auf diese Zeit lautet. In Frankreich wäre es dagegen unhöflich, um Punkt 20 Uhr an der Tür zu klingeln. Üblich ist es eben, etwa eine Viertelstunde später zu kommen.

Der Aspekt der „Monochronie“: Eine Aufgabe allein und gründlich über eine bestimmte Zeitspanne auszuführen, ist in nordischen Kulturen üblich, während südliche Kulturen eher „polychronisch“ funktionieren: Mehrere Aufgaben werden gleichzeitig in Angriff genommen, Störungen und Unterbrechungen durch die Umgebung werden kaum als negativ empfunden. Unterbrechen der Gesprächspartner(innen) in der Diskussion gilt dort nicht als unhöflich, sondern eher als Interessenbekundung.

Im Augenblick leben ist eher in südländischen Kulturen üblich, während nordische Kulturen sich meistens in die Zukunft projizieren und eventuelle Schwierigkeiten zu antizipieren versuchen. Es ist beispielsweise durchaus denkbar, dass sich zwei nach Deutschland reisende Drogenkuriere aus Nigeria während des Fluges damit begnügen, den Film im Bordkino anzuschauen, ohne sich über ihre Ankunft in Deutschland und eventuell zu erwartende Schwierigkeiten näher zu unterhalten. Dies erscheint vielen deutschen Richter(inne)n zunächst als unglaublich und kann leicht zu Missverständnissen in der Aussagenbewertung führen.

\section{Wahrung des Gesichts (Schamgefühl)}

Schamgefühle oder Beleidigungen sind ebenfalls kulturbedingt. Ein afrikanischer Angeklagter, der die Dolmetscherin als Respektsperson achtete, teilte seinem Verteidiger mit, dass er die lange Zeit verneinte Schuld in Anwesenheit der Dolmetscherin aus Scham nicht eingestehen wolle.

\section{Familie, soziale Hierarchie}

Die Unterschiede der Familienbindungen und soziale Hierarchie sind ebenfalls richtig zu interpretieren. Mittlerweile ist bekannt, dass ein Bruder auf dem afrikanischen Kontinent nicht unbedingt auf gemeinsame Eltern hindeutet. Aufgrund der herrschenden sozialen Hierarchie kann ferner die sehr einflussreiche Rolle eines (einer) als Laiensprachmittler fungierenden Sprachkundigen in einer bestimmten ethnischen Gemeinde insbesondere hinsichtlich der Frauenintegration besonders kritisch werden.

\section{Ernährung, Esskultur, Gesundheit}

Auf Unterschiede der Ernährung und Esskultur braucht hier nicht näher eingegangen zu werden, da diese mittlerweile recht allgemein bekannt sind, obwohl die Auseinandersetzung mit den religiösen Bräuchen (Fastenzeiten und der Begriff der unreinen bzw. reinen Nahrungsmittel) nützlich ist. Kulturelle Unterschiede in Bezug auf Krankheiten sind hier noch als interessant zu erwähnen. In europäischen Nachbarländern wie Deutschland, Frankreich und dem Vereinigten Königreich ist das Bestehen unterschiedlicher "Lieblingssymptome“ festzustellen. Nach einem üppigen Mahl behaupten Franzosen zum Beispiel, an Leberschmerzen (crise de foie) zu leiden, Deutsche beschweren sich über ihren Magen, während Engländer eher auf Darmbeschwerden (Bowel), natürlich sehr diskret, hinweisen. Wie kann ein(e) deutsche(r) Richter(in) einen Hinweis auf Leberschmerzen ohne diese kulturelle Erfahrung richtig interpretieren und bewerten? Meistens fragt er (sie), ob diese Erklärung von einem (einer) Arzt (Ärztin) bestätigt wurde und meistens ist dies eben nicht der Fall. Daraufhin kann die Glaubwürdigkeit der Aussage wahrscheinlich in Frage gestellt werden. Eine wortgetreue Übersetzung ist hier ohne kulturellen Hinweis nur irreführend.

\section{Geschichte, Traditionen, Politik, Institutionen}

Diese Dimensionen sind ohne längere Erklärung sicherlich leicht nachvollziehbar. Jedoch kann ein Beispiel mögliche Folgen ihrer Unkenntnis sie noch prägnanter erscheinen lassen. Vor dem Fall der Mauer wurde der bundesdeutsche Nationalfeiertag am 17. Juni in Erinnerung an den Berliner Volksaufstand 1953 eher mit Trauer im Andenken an die zahlreichen Opfer begangen. In einem großen Pariser Restaurant wurde an einem 17. Juni deutschen Gästen überschwänglich zu ihrem

4 Kumbruck, Christel/Derboven, Wibke, Interkulturelles Training zur Förderung interkultureller Kompetenzen in der Arbeit, 2. erweiterte und überarbeitete Auflage, Wiesbaden 2009; Lüsebrink, Hans-Jürgen, Interkulturelle Kommunikation: Interaktion, Fremdwahrnehmung, Kulturtransfer, Stuttgart/Weimar 2009. 
Nationalfeiertag gratuliert, ihnen wurde sogar viel Spaß bei den Feierlichkeiten gewünscht. Warum eine solche Taktlosigkeit? Ganz einfach: In der Vorstellung eines französischen Bürgers kann ein Nationalfeiertag nur fröhlich wie der 14. Juli mit Straßenmusik, Bällen und Feuerwerk sein.

\section{Sprachliche und soziokulturelle Kluft}

Zusätzlich zu den eben skizzierten interkulturellen Dimensionen sind andere Faktoren wie die oft innerhalb derselben Sprache bestehenden kulturellen Nuancen zu berücksichtigen: Englisch wird weltweit gesprochen, jedoch unterscheidet sich zum Beispiel das in Indien praktizierte Englisch sehr von dem in Kanada oder in Australien gesprochenen Englisch. Ähnliches gilt für das Französische, das Spanische oder das Deutsche.

Darüber hinaus darf auch die Schwierigkeit der sozialen Distanz nicht übersehen werden. Ein Problem, das innersprachlich schon besteht und zwischen zwei Sprachen einen kumulativen Effekt erreicht. Französisch dolmetschen zum Beispiel zwischen einem Fernfahrer und einem deutschen Juristen zum Beispiel bringt oft vielschichtige Probleme mit sich.

Die Sensibilisierung auf diese sprachgebundene Problematik ist essenziell. Diese Sensibilisierung sollte drei ineinander verzahnte Kernziele erreichen:

- Richtig verstehen: D. h. insbesondere imstande zu sein, mögliche kulturbedingte Missverständnisse zu erahnen. Ohne richtiges Verstehen, ohne Vermeidung eventueller kultureller Missverständnisse, schlagen Kommunizieren und Bewerten fehl. Eine interkulturelle Sensibilität lässt auch ein für die Wahrheitsfindung sinnvolles Zweifeln bei der Anwendung der eigenen Werte zu.

- Richtig kommunizieren, um selbst richtig verstanden zu werden, um dadurch die gewünschte relevante Information zu erhalten.

- Richtig bewerten ist wiederum nur möglich, wenn die Information richtig verstanden wurde.

\section{Welche interkulturellen Kompetenzen für die Justiz?}

Diese kurz skizzierten Kernmerkmale der interkulturellen Kommunikation sind hier lediglich Denkanstöße. Die aufgeführten Beispiele könnten als Checkliste im Falle von merkwürdig erscheinendem Verhalten oder Aussagen von Ausländer(inne)n zu Hilfe genommen werden.

Ein gründliches Verständnis für eine fremde Kultur ist ohne Kenntnis der entsprechenden Landessprache kaum möglich. Berücksichtigt man die Vielzahl der im Rechtsverkehr heutzutage in Erscheinung tretenden Sprachen, wäre es unrealistisch, von den Justizangehörigen den Erwerb von Kenntnissen in mehreren dieser Sprachen verlangen zu wollen.

Aus diesen Gründen sind der Einsatz von qualifizierten Dolmetscher(inne)n und Sprachsachverständigen und das Erlernen der richtigen Zusammenarbeit mit ihnen unentbehrlich. Dies beginnt mit dem direkten Ansprechen der der Gerichtssprache nicht mächtiger Ausländer(innen) zur Vermeidung jeder Konfusion. Die Formel „Fragen Sie ihn (sie) mal ..." sollte daher absolut vermieden werden. Der (die) Dolmetscher(in) spricht selbst in der ersten Person, wenn er (sie) im Namen des (der) Befragten antwortet und in der dritten Person in seinem (ihrem) eigenen Namen.

\section{Nur qualifizierte Dolmetscher(innen) einsetzen}

Im Sinne eines fairen Verfahrens ist der Einsatz qualifizierter Dolmetscher(innen) unabdingbar. Ein(e) qualifizierte(r) Dolmetscher(in) beherrscht Dolmetschtechniken, die entsprechend der Verfahrensphasen für eine optimale Kommunikation eingesetzt werden:

v Vom-Blatt-Übersetzen für das exakte und schnelle Übersetzen von in der Sitzung überreichten Schriftsätzen.

- Konsekutivdolmetschen ohne Notizen und mit einer besonderen Notizentechnik, für das zuverlässige Dolmetschen von Aussagen in die Gerichtssprache ohne unnatürliche und störende Unterbrechung der Vernommenen.

- Simultandolmetschen, meist in der Variante des Flüsterdolmetschens, für die vollständige Verdolmetschung meist in die Sprache des (der) Angeklagten (Antragstellers (Antragstellerin) in anderen Verfahrensarten).

- Ferner verfügt der (die) qualifizierte Dolmetscher(in) über die notwendigen forensischen Kenntnisse. Für das richtige Dolmetschen ist das Verständnis über den Verfahrensablauf wichtig.

- Schließlich unterzieht sich der (die) qualifizierte Gerichtsdolmetscher(in) einem strengen Berufsethos, der unter anderem von den Grundsätzen der europäischen Menschenrechtskonvention abgeleitet wird. Die Verdolmetschung soll den (die) Sprachunkundige(n) möglichst in die Lage eines (einer) der Gerichtssprache mächtigen Bürgers (Bürgerin) versetzen.

- Wie bei anderen Spielarten des Dolmetschens erhalten ferner die Schweigepflicht und die Neutralität einen wichtigen Platz.

Die Bedarfsträger der Justiz sollten auf dieser Grundlage die Qualifikation und Leistungen des (der) eingesetzten Dolmetschers (Dolmetscherin) bewerten lernen und im Sinne eines fairen Verfahrens keine Zugeständnisse diesbezüglich eingehen.

\section{Spannungsfeld zwischen Quellen- und Zielkultur}

Oft wird der Dolmetsch- bzw. Übersetzungsprozess nicht richtig verstanden: Nicht selten wird der (die) Dolmetscher(in) mit folgender Aufforderung konfrontiert: „Sie sollen ja nur wörtlich übersetzen. “ Nur wortgetreu übersetzen ist nur bei eindeutigen (monorefenziellen) Begriffen möglich und legitim (Zahlen, Ortsnamen, technische Fachbegriffe). Die Schwierigkeiten beginnen jedoch schon bei der Übersetzung von Institutionen, angefangen bei den Gerichtsbezeichnungen. Wie ist zum Beispiel der Begriff „Landgericht“ für einen zentralistisch regierten Staat verständlich zu übersetzen? Fachbegriffe wie „Pflegeversicherung“ oder „Tagessatz“ waren lange Jahre nur in Deutschland bekannt; eine wörtliche Übersetzung war zwar möglich, jedoch ohne Erklärung unbegreiflich. 
Um sich die Sinnlosigkeit einer rein wörtlichen Übersetzung vor Augen zu führen, sollte man eine Urkunde oder ein Urteil zum Beispiel durch ein Online-Übersetzungsprogramm durchführen lassen.

Sinngetreu (und keinesfalls sinngemäß) übersetzen ist dagegen realistisch: Dabei wird die Aussage dem Erfassungsvermögen der Zuhörer entsprechend in der Zielsprache formuliert. Tonfall und Gestik sind dabei auch zu berücksichtigen. Ein einfaches Beispiel darf hier eingeführt werden: Wenn ein Norddeutscher mit einem bestimmten Tonfall sagt „Der Film war gut!“ wird diese Aussage häufig mit „sehr gut“ in die französische Sprache korrekt übersetzt.

\section{Ethische Problematik der Rednerintention}

Im Rahmen der interkulturellen Kommunikation und bei einer sinngetreuen Verdolmetschung ist die Loyalität zur Rednerabsicht eine wichtige Komponente; damit ist hauptsächlich die Absicht des (der) Redners (Rednerin) gemeint.

Zwei Fälle können als Beispiel hier erwähnt werden: Im Rahmen eines europäischen Juristentreffens ist ein Redner für die Zielkulturen (unabsichtlich) grob, indem er die Begrüßung oder das Danken in der Eile vergisst. Der Dolmetscher kann sich durchaus nach den Absichten des Redners richten.

In einem anderen Fall ist ein Redner, ein ukrainischer Untersuchungshäftling, für die eigene und andere Kultur (absichtlich) grob, indem er gegenüber dem Dolmetscher eine abfällige Bemerkung über die vernehmende Staatsanwältin macht: „Was hast du hier für eine Schlampe herangeschleppt?" Bei einer so deutlichen Absicht hat der Dolmet- scher keinen Grund, die Mitteilung in seiner Übersetzung zu mildern.

In beiden und in ähnlichen Fällen übernimmt der (die) Dolmetscher(in) eine ethische Verantwortung.

\section{Zusammenfassung und Appell}

In dieser Übersicht wurde versucht, die Wichtigkeit einer Sensibilisierung der Justiz für multikulturelle Unterschiede aufzuzeigen, indem die Kernaspekte kurz skizziert wurden. Diese Kernaspekte könnten in einer Art Checkliste zusammengefasst werden, die im Falle von Verständnisschwierigkeiten zu Hilfe gezogen werden könnte. Wichtig dabei wäre, das Aufkommen von Fehlinterpretationen zu antizipieren und zu vermeiden, indem Dolmetscher(innen) als Kultur- und Sprachsachverständige gleich zu Rate gezogen werden.

Bei den stets wechselnden Migrationswellen kann von den Justizberufen nicht erwartet werden, dass sie sich auf so viele Kulturen und Sprachen spezialisieren können. Die kulturelle Öffnung der Justiz könnte jedoch durch eine systematische Pluridisziplinarität erreicht werden, das heißt, durch die $\mathrm{Zu}$ sammenarbeit mit juristischen Dolmetschern und Übersetzer(inne) $n$ aber auch mit deren Ausbilder(inne)n.

Im Sinne eines fairen Verfahrens, der würdigen Behandlung der Parteien und nicht zuletzt zur Förderung der Wahrheitsfindung sind der Einsatz qualifizierter Dolmetscher(innen) und die korrekte und kompromisslose Beurteilung ihrer Leistung dringend anzustreben. Seit den Nürnberger Prozessen sind diese Grundsätze im Rahmen der internationalen Gerichtsbarkeiten Tradition geworden ist. Warum sollten die nationalen Gerichte diese Praxis nicht anstreben?

\section{Rezension: Geschlecht im Recht. Eine fortbestehende Herausforderung}

\author{
Beate Rudolf (Hrsg.) \\ Querelles. Jahrbuch für Frauen- und Geschlechterforschung (Re- \\ daktion: Anita Runge) \\ Bandnummer: 14/2009 \\ Preis: 24,00 Euro (D), 24,70 Euro (A), 36,90 CHF (CH) \\ Einband: broschiert, Format: $14 \times 22,2 \mathrm{~cm}$ \\ 304 Seiten mit 7 Abbildungen \\ ISBN: 978-3-8353-0448-2
}

Mit dem Jahrbuch Querelles 2009 verfügt die Rechtswissenschaft über ein weiteres wichtiges Buch, das versucht die Erkenntnisse der Frauen- und Geschlechterstudien und ihrer juristischen Rezeption einem breiteren Publikum zugänglich zu machen. Der Herausgeberin Beate Rudolf ist es gelungen, viele namhafte Wissenschaftlerinnen aus dem Bereich der feministischen Rechtswissenschaften für einen Beitrag zu gewinnen. Allen Beiträgen geht es um ein Sichtbarmachen der Einflüsse

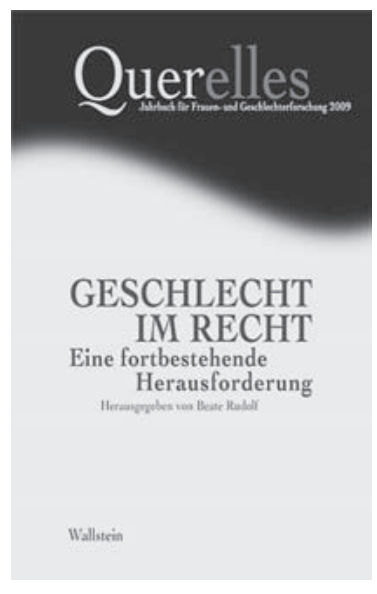

der Frauen und Geschlechterforschung auf die jeweiligen Rechtsgebiete, wenngleich die Berücksichtigung des Standes dieser Forschung unterschiedlich intensiv ausfällt. Leider bleiben das Vertrags-, Beamten- und weitgehend das Familienrecht unbehandelt. Man mag die Vielfalt der Ansätze in den verschiedenen Beiträgen, die auch nicht immer ganz frei von Widersprüchen sind, kritisieren. Viel eher ist aber zu honorieren, dass genau hierdurch das von Susanne Baer in ihrem einleitenden Beitrag („Entwicklung und Stand feministischer Rechtswissenschaft in Deutschland“) dargelegte Selbstverständnis der feministischen Rechtswissen- 